\title{
Validade de medidas antropométricas autorreferidas em adolescentes: sua relação com percepção e satisfação corporal
}

\author{
Anthropometric self-reported measures: relationship to body \\ image perception and satisfaction among adolescents
}

Adriane Bueno Marangoni', Helymar da Costa Machado², Maria Aparecida Zanetti Passos', Mauro Fisberg', Isa de Pádua Cintra'

\section{RESUMO}

Objetivo: Verificar a validade da classificação do estado nutricional e da estatura para idade quando utilizadas massa corporal e estatura autorreferidas por adolescentes, bem como relacionar essa validade com percepção e com satisfação corporal. Métodos: 304 adolescentes antes de terem suas medidas de massa e estatura aferidas autorreferiram essas medidas, foram questionados sobre a percepção de seu corpo e a classificação da imagem corporal foi obtida com o uso de uma escala de silhuetas corporais. Também foram aplicados testes específicos para avaliar a satisfação corporal e o comportamento alimentar. Resultados: As classificações feitas com base nos dados autorreferidos e aferidos tiveram concordância alta $(K=1,00)$ para a classificação da estatura por idade e moderada $(K<0,75)$ para a classificação de índice de massa corporal. No entanto, adolescentes que se consideravam "abaixo do peso" subestimaram o índice de massa corporal autorrelatado e, os que se percebiam como "gordos", o superestimaram. As meninas e os que se classi-

\section{Palavras-chave}

Adolescente, peso corporal, estatura, validade, imagem corporal. ficaram como obesos tenderam a subestimar o índice de massa corporal, e os meninos, a superestimá-lo. Conclusão: Os adolescentes autorrelataram suas medidas de massa e estatura de maneira adequada. No entanto, o sexo, a percepção e a classificação da imagem corporal foram variáveis que influenciaram na obtenção de respostas não válidas.

\section{ABSTRACT}

Objective: To assess the reliability of the classification for nutritional status and height-for-age when using self-reported weight and height and its relationships with self-reported data of body image perception and satisfaction among adolescents. Methods: In this retrospective study, 304 adolescents before they had measured their height and weight, they self-reported these measures, they were asked about their body shape perception and body image classification was obtained by using a body silhouette scale. It was also used specific tests to assess body satisfaction and food behavior. Results: It was found full agreement between height-for-age classification using self-reported and measured height $(k=1.00)$ and an intermediate level of agreement between body mass index calculated with self-reported and measured data $(k<0.75)$. However,

1 Universidade Federal de São Paulo (Unifesp), Centro de Atendimento e Apoio ao Adolescente, Departamento de Pediatria. 2 Universidade Estadual de Campinas (Unicamp), Faculdade de Ciências Médicas.

Endereço para correspondência: Adriane Bueno Marangoni Rua Botucatu, 715 - Vila Clementino - 04023-062 - São Paulo, SP

Email: adrianemarangoni@gmail.com 


\section{Keywords}

Adolescent, body weight, body height, validity, body image. adolescents who perceived themselves as "skinny" underestimated their self-reported body mass index whereas those who perceived themselves as "fat" overestimated it. Those who perceived themselves as fat in the silhouette scale underestimated their body mass index. Gender was also a factor for unreliable answers, as females tended to underestimate their self-reported body mass index and males to overestimate it. Conclusion: The adolescents adequately self-reported weight and height information. However, body shape perception and body image classification were factors affecting answer reliability.

\section{INTRODUÇÃO}

O uso de medidas autorreferidas, como massa e estatura, é comumente utilizado em pesquisas em decorrência da praticidade e do baixo custo. Nessa estratégia, o profissional não realiza a tomada das medidas utilizando-se de balança e estadiômetro; são os próprios avaliados que relatam os valores de suas medidas ${ }^{1,2}$. Apesar das facilidades para a coleta de dados, tanto entre as mulheres quanto entre os homens, independentemente de serem adultos ou adolescentes, há a tendência de subestimação da massa corporal, principalmente entre as mulheres e os indivíduos obesos ${ }^{1,3}$.

No que diz respeito ao autorrelato de estatura, percebe-se, por sua vez, uma superestimação dessa medida entre os homens e indivíduos de baixa estatura. Ou seja, há uma tendência de as pessoas alterarem os valores em busca de uma estética corporal socialmente valorizada ${ }^{3}$.

De acordo com Pregnolato et al.2., embora não haja consenso na literatura, a maioria dos estudos demonstra superestimação de estatura e subestimação de massa corporal, em ambos os sexos; no entanto, é preciso considerar aspectos sociais, culturais, étnicos, etários e de gênero, antes de se optar pelas medidas autorreferidas como estratégia para avaliar o estado nutricional de grupos populacionais.

No caso específico dos adolescentes, a percepção de que o corpo está se transformando pode ocorrer de forma tranquila ou gerar insatisfação, contribuindo para que o adolescente se autoavalie de maneira distorcida ou inadequada4.

O objetivo do presente estudo foi o de verificar a validade da classificação do estado nutricional e da classificação de estatura para idade quando utilizadas massa corporal e estatura autorreferidas por adolescentes, bem como relacionar a validade dos dados autorreferidos com a percepção e com a satisfação corporal.

\section{MÉTODOS}

Trata-se de um estudo transversal, que ocorreu em 2003 na região central da cidade de Cotia (SP), na qual foi utilizada uma amostra por conveniência. Foram contatadas cinco escolas da região central de Cotia (sendo que destas, três eram públicas e duas particulares), e aquelas que aceitaram participar da pesquisa passaram a fazer parte do estudo.
Os adolescentes tiveram suas medidas de massa e estatura aferidas logo após o autorrelato destas. A aferição das medidas seguiu a padronização proposta por Jelliffe e pela World Health Organization ${ }^{5,6}$ e todos os antropometristas receberam treinamento específico, de forma a minimizar possíveis erros na coleta dos dados.

A percepção da imagem corporal foi obtida por autoavaliação, com o uso de uma escala de silhuetas corporais (1 a 9) em que se estabelecem quatro categorias: baixo peso (1), eutrofia (2 a 5), sobrepeso (6 e 7), e obesidade (8 e 9)7.

$\mathrm{O}$ adolescente também foi questionado sobre qual era a percepção que ele tinha em relação à sua massa corporal. Como resposta a esse questionamento, o adolescente deveria escolher uma das seguintes alternativas: "muito abaixo do peso", "abaixo do peso", "médio", "gordo" e "muito gordo".

Para análise da satisfação corporal, foi aplicado o Body Shape Questionnaire (BSQ) ${ }^{8}$, conforme versão traduzida para o português por Cordás e Castilho ${ }^{9}$ do original de Cooper et al. validada por Conti et al..$^{10}$.

$\mathrm{Na}$ análise estatística foram utilizados os testes a seguir, levando-se em conta o fato de as variáveis do estudo em questão não terem distribuição normal: coeficiente de correlação intraclasse, coeficiente de correlação de Spearman e teste de Wilcoxon para análises de concordância entre os valores de massa corporal, estatura e índice de massa corporal (IMC) aferidos e referidos; coeficiente kapa, teste de McNemar e teste de simetria de Bowker para análises de concordância entre classificação do estado nutricional a partir de medidas aferidas e medidas autorreferidas; e teste exato de Fisher para comparação entre concordância de IMC aferido e referido com variáveis como percepção em relação a massa, insatisfação corporal e classificação da imagem corporal. Em relação ao uso do coeficiente de correlação intraclasse, foi seguida a metodologia proposta por Streiner e Norman"1.

O nível de significância adotado para os testes estatísticos foi de $5 \%$, ou seja, $p<0,05$.

O software utilizado para análise estatística foi The SAS System for Windows (Statistical Analysis System), versão 8.02. SAS Institute Inc, 1999-2001, Cary, NC, USA ${ }^{15}$.

Este estudo está em conformidade com princípios éticos para pesquisas envolvendo seres humanos, em conformidade com a Resolução 196/96 do Conselho Nacional de Medicina, e foi aprovado pelo Comitê de Ética do Conselho de Pós-graduação e Pesquisa da Universidade Federal de São Paulo (Unifesp) sob o protocolo n. ${ }^{\circ} 0977 / 03$. 


\section{RESULTADOS}

Das cinco escolas contatadas, apenas três aceitaram participar da pesquisa, sendo duas particulares e uma pública.

Dos 304 adolescentes que participaram do presente estudo, 59,9\% ( $n=182)$ estudavam em escola particular e $51 \%$ $(n=155)$ eram do sexo masculino. Em relação à idade, 30,3\% $(n=92)$ tinham entre 10 e 12 anos, 48,3\% ( $n=147)$ entre 13 e 15 anos e $21,4 \%(n=65)$ entre 16 e 18 anos (média $=14,38$ $\pm 1,85$ anos).
Verificaram-se alta concordância (CCl) e correlação (Spearman) entre os valores aferidos e referidos para as três medidas (massa corporal, estatura e IMC) (Figura 1).

Ao avaliar a relação entre as medidas aferidas e referidas, não foi encontrada diferença significante entre os valores da massa corporal $(55,01 \mathrm{~kg} \pm 13,77$ versus $55,19 \mathrm{~kg} \pm 13,59 ; \mathrm{p}=0,161) \mathrm{e}$ do IMC $\left(20,82 \mathrm{~kg} / \mathrm{m}^{2} \pm 3,88\right.$ versus $\left.20,71 \mathrm{~kg} / \mathrm{m}^{2} \pm 3,70, p=0,162\right)$, porém houve diferença significante entre estatura referida e aferida - maiores valores de estatura referida do que estatura aferida (162,34 $\mathrm{cm} \pm 10,53$ versus $161,66 \mathrm{~cm} \pm 10,73 ; p<0,001)$.

\section{Massa corporal:}

CCI: 0,971; IC95\% CCl: $(0,964 ; 0,977) ; \mathbf{p}<\mathbf{0 , 0 0 1}$

Coeficiente de Correlação de Spearman:

$r=0,9711 ; p<0,0001 ; n=304$

Teste de Wilcoxon para amostras relacionadas: $p=0,161$

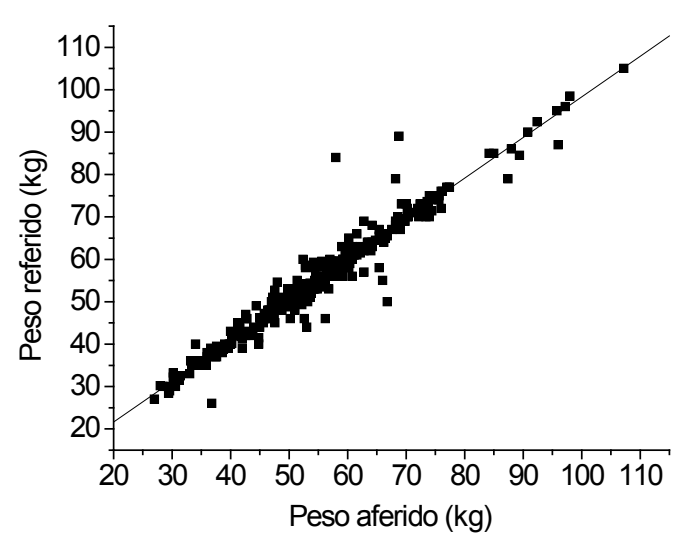

\section{Estatura:}

CCI: 0,967; IC95\% CCl: $(0,956 ; 0,974) ; \mathbf{p}<\mathbf{0 , 0 0 1}$

Coeficiente de Correlação de Spearman $r=0,9670 ; \mathbf{p}<\mathbf{0 , 0 0 0 1 ;} n=304$

Teste de Wilcoxon para amostras relacionadas:

$\mathbf{p}<0,001$

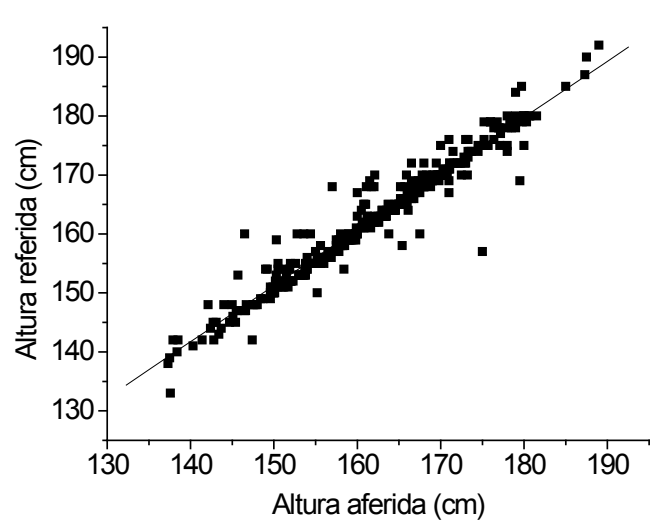

IMC:

ICC: 0,930; IC95\% ICC: $(0,913 ; 0,944) ;$ P $<\mathbf{0 , 0 0 1}$

Coeficiente de Correlação de Spearman $r=0,9245 ; \mathbf{p}<\mathbf{0 , 0 0 0 1 ;} n=304$

Teste de Wilcoxon para amostras relacionadas:

$p=0,162$

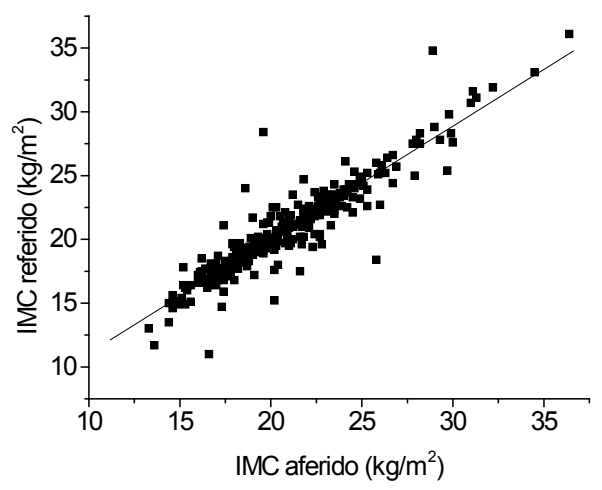

CCl: coeficiente de correlação intraclasse; IC95\%: intervalo de confiança de 95\%; IMC: índice de massa corporal.

Figura 1. Análise de concordância entre medidas antropométricas aferidas e referidas. 
As tabelas 1 e 2, por sua vez, apresentam as análises de concordância entre a classificação de estado nutricional feita com base em dados aferidos e referidos. Pelos resultados, verifica-se alta concordância entre a classificação da estatura por idade feita com base na estatura aferida e na referida ( $K$ $=1,00$ ) (Tabela 1), e concordância moderada entre classificação de IMC aferido e referido $(K<0,75)$, sem diferença significante entre as medidas (Tabela 2).

Tabela 1. Análise de concordância entre medidas de estatura aferidas e referidas

\begin{tabular}{lccc}
\hline \multirow{2}{*}{$\begin{array}{l}\text { Classificação da estatura/idade } \\
\text { (aferida) }\end{array}$} & \multicolumn{3}{c}{ Classificação estatura/idade (referida) } \\
\cline { 2 - 4 } & Baixa estatura & Estatura adequada & Total (n) \\
\hline Baixa estatura & $1,0 \%$ & $0,0 \%$ & 3 \\
Estatura adequada & $0,0 \%$ & $99,0 \%$ & 301 \\
Total (n) & 3 & 301 & 304 \\
\hline
\end{tabular}

Teste de McNemar: $p=1,000 ;$ Kapa: 1,000; IC95\% $(1,000 ; 1,000)$.

Tabela 2. Análise de concordância entre estado nutricional aferido e referido

\begin{tabular}{|c|c|c|c|c|c|}
\hline \multirow{2}{*}{$\begin{array}{l}\text { Classificação IMC } \\
\text { aferido }\end{array}$} & \multicolumn{4}{|c|}{ Classificação IMC referido } & \multirow{2}{*}{ Total (n) } \\
\hline & Magreza & Eutrofia & Sobrepeso & Obesidade & \\
\hline Magreza & $1,3 \%$ & $0,7 \%$ & $0 \%$ & $0 \%$ & 6 \\
\hline Eutrofia & $1,3 \%$ & $66,5 \%$ & $3,6 \%$ & $0,3 \%$ & 218 \\
\hline Sobrepeso & $0 \%$ & $3,9 \%$ & $16,5 \%$ & $0,3 \%$ & 63 \\
\hline Obesidade & $0 \%$ & $0,3 \%$ & $1,6 \%$ & $3,7 \%$ & 17 \\
\hline Total (n) & 8 & 217 & 66 & 13 & 304 \\
\hline
\end{tabular}

Teste de Simetria: $p=0,760 ;$ Kapa: 0,740; IC95\% (0,660; 0,821).

IMC: índice de massa corporal.
Foi verificado entre os adolescentes com IMC aferido menor, igual e maior do que o referido a relação que eles apresentavam com a distribuição do BSQ (sem insatisfação, insatisfação leve, moderada e grave); da percepção do peso corporal (muito abaixo, abaixo, médio, gordo, muito gordo) e da imagem corporal (baixo peso, eutrofia, sobrepeso e obesidade), tendo sido observado que tanto a percepção como a imagem corporal são fatores importantes a serem considerados (Tabela 3).

Verificou-se que, entre os adolescentes do sexo masculino e feminino, 1,94\% e 2,01\% eram magros; $20 \%$ e $21,48 \%$ estavam com sobrepeso e $5,16 \%$ e $6,04 \%$, respectivamente, eram obesos. No entanto, ao se fazer a distribuição entre os IMC aferido e referido, de acordo com o sexo, observou-se que há uma distorção entre os valores de IMC (Tabela 4).

Tabela 4. Relação entre IMC aferido e referido de acordo com o sexo

\begin{tabular}{lccc}
\hline & \multicolumn{3}{c}{ IMC } \\
\cline { 2 - 4 } & $\begin{array}{c}\text { Afe }<\text { Ref } \\
(\%)\end{array}$ & $\begin{array}{c}\text { Afe }=\text { Ref } \\
(\%)\end{array}$ & $\begin{array}{c}\text { Afe }>\text { Ref } \\
(\%)\end{array}$ \\
\hline Sexo masculino & $85,35(n=14)$ & $48,85(n=128)$ & $52(n=13)$ \\
Sexo feminino & $17,65(n=3)$ & $51,15(n=134)$ & $48(n=12)$ \\
\hline
\end{tabular}

Teste qui-quadrado: $p=0,028$

IMC: índice de massa corporal.

Tabela 3. Relação entre IMC aferido e referido de acordo com o BSQ, BITE e EAT, percepção do próprio peso e imagem corporal

\begin{tabular}{|c|c|c|c|c|}
\hline & \multicolumn{3}{|c|}{ Índice de Massa Corporal } & \multirow[b]{2}{*}{ Valorp } \\
\hline & $\begin{array}{c}\text { Afe }<\text { Ref } \\
(\%)\end{array}$ & $\begin{array}{c}\text { Afe }=\text { Ref } \\
(\%)\end{array}$ & $\begin{array}{c}\text { Afe }>\text { Ref } \\
(\%)\end{array}$ & \\
\hline BSQ - sem insatisfação $(n=179)$ & $58,82(n=10)$ & $61,57(n=157)$ & $48(n=12)$ & 0,519 \\
\hline BSQ - insatisfação leve $(n=60)$ & $23,53(n=4)$ & $20(n=51)$ & $20(n=5)$ & \\
\hline BSQ - insatisfação moderada $(n=39)$ & $17,65(n=3)$ & $11,76(n=30)$ & $24(\mathrm{n}=6)$ & \\
\hline BSQ - insatisfação grave $(n=19)$ & 0 & $6,67(n=17)$ & $8(n=2)$ & \\
\hline Percepção - muito abaixo ( $n=15)$ & $5,88(n=1)$ & $3,91(n=10)$ & $17,39(n=4)$ & 0,013 \\
\hline Percepção - abaixo (n= 46) & $5,88(n=1)$ & $15,63(n=40)$ & $21,74(n=5)$ & \\
\hline Percepção - médio ( $n=175$ ) & $47,06(n=8)$ & $62,11(n=159)$ & $34,7(n=8)$ & \\
\hline Percepção - gordo $(\mathrm{n}=46)$ & $29,41(n=5)$ & $14,45(n=37)$ & $17,39(n=4)$ & \\
\hline Percepção - muito gordo ( $n=14)$ & $11,76(n=2)$ & $3,91(n=10)$ & $8,7(n=2)$ & \\
\hline Imagem corporal - baixo peso $(n=12)$ & $6,67(n=1)$ & $4,74(n=10)$ & $4,76(n=1)$ & 0,009 \\
\hline Imagem corporal - eutrofia $(n=122)$ & $60(n=9)$ & $51,18(n=108)$ & $23,81(n=5)$ & \\
\hline Imagem corporal - sobrepeso $(\mathrm{n}=86)$ & $33,33(n=5)$ & $35,07(n=74)$ & $33,33(n=7)$ & \\
\hline Imagem corporal - obesidade $(n=27)$ & 0 & $9(n=19)$ & $38,1(n=8)$ & \\
\hline
\end{tabular}

Teste exato de Fisher.

BSQ: Body Shape Questionnaire 


\section{DISCUSSÃO}

Os adolescentes do presente estudo autorrelataram suas medidas de massa e estatura de maneira adequada, uma vez que a estatura referida, mesmo tendo se mostrado maior do que a aferida, não foi o suficiente para influenciar de forma significante na classificação de estatura para idade. A classificação de estado nutricional feita com base nos dados referidos e aferidos, por sua vez, apresentou concordância moderada, mas sem diferenças estatisticamente significantes entre as medidas.

No entanto, foi possível perceber uma tendência ao relato não válido das medidas em algumas situações. No caso das meninas, por exemplo, foi possível perceber que elas tendiam a subestimar OIMC, enquanto os meninos tendiam a superestimá-lo. Não foi encontrada relação estatisticamente significante entre classificações obtidas pelo teste BSQ com discrepância entre IMC aferido e referido, no entanto, pôde ser verificado que em pessoas com insatisfação corporal moderada e grave pelo BSQ houve uma maior tendência de subestimação no autorrelato do IMC. A percepção da massa corporal dos participantes do presente estudo, por sua vez, interferiu de forma estatisticamente significante na discrepância do IMC, calculado com base em dados referidos e aferidos, quando essa percepção estava nos limites da classificação, tanto no nível superior (gordo e muito gordo) quanto no limite inferior (abaixo ou muito abaixo do peso ideal). Pessoas que se consideravam abaixo do peso tenderam a subestimar o seu IMC quando no autorrelato das medidas de massa e estatura. Já aqueles que se percebiam como sendo gordo ou muito gordo tenderam a superestimar seu IMC. A classificação da imagem corporal mostrou também uma relação estatisticamente significante com a discrepância encontrada entre IMC feito com base nos dados aferidos e autorrelatados. No entanto, essa relação estatisticamente significante foi encontrada apenas na classificação do limite superior (obesidade). Aqueles que se classificaram como obesos tiveram valores inferiores de IMC autorrelatados quando comparados aos valores reais.

Apesar de vários estudos não terem mostrado concordância entre dados antropométricos aferidos e referidos $^{2,3,13-15}$, alguns resultados antagônicos puderam ser verificados na literatura. Em estudo realizado no Rio de Janeiro (RJ) ( $n=3.703)$, por exemplo, os autores verificaram alta concordância entre a aferição e a informação da massa e da estatura ${ }^{16}$, e em estudo realizado com 943 indivíduos adultos da população mexicana ${ }^{17}$, as medidas autorreferidas também se mostraram válidas para a avaliação do estado nutricional.

Alguns estudos têm verificado uma maior tendência do sexo feminino a não autorrelatar suas medidas antropométricas de forma válida 2,14,18. Em um estudo realizado com 944 indivíduos na cidade de Santos (SP)², por exemplo, quando comparados os dados autorreferidos com os referidos, foi possível verificar que a massa corporal foi subestimada e a estatura, superestimada. No entanto, essas diferenças só foram evidenciadas no sexo feminino ( $n=549$, com média de idade 28,3 $\pm 11,0$ anos). Um estudo realizado em Florianópolis $(\mathrm{SC})^{14}$, com escolares de 15 a 18 anos $(\mathrm{n}=867)$, também verificou valores mais discrepantes entre os dados autorreferidos por indivíduos do sexo feminino. Os resultados mostraram a subestimativa da massa corporal e a superestimativa da estatura quando no uso de medidas autorreferidas, resultando em subestimação do IMC de $0,07 \mathrm{~kg} / \mathrm{m}^{2}$ para os rapazes e 0,56 kg/m² para as moças. Também na França foi encontrada uma maior tendência ao sub-relato de IMC em mulheres (sub-relato de IMC de $0,29 \mathrm{~kg} / \mathrm{m}^{2}$ para homens e $0,44 \mathrm{~kg} / \mathrm{m}^{2}$ em mulheres $)^{18}$.

No atual estudo, foi encontrada apenas no sexo feminino uma tendência ao sub-relato, pois os meninos, por sua vez, tenderam a superestimar o IMC autorrelatado. Isso pode indicar padrões estéticos vigentes que são diferentes para os sexos - enquanto para mulher o padrão estético é a magreza, para os homens um corpo musculoso possa ser o padrão desejado. Tal padrão não foi encontrado entre adolescentes dos Estados Unidos, pois, em estudo realizado no país com 2.032 estudantes de segundo grau ${ }^{15}$, subestimativas de IMC feito com base nos dados autorrelatados foram verificadas em ambos os sexos. Já um estudo realizado na Austrália, com meninas de 11 a 18 anos de idade, mostrou que aquelas que tinham maior IMC tendiam a um maior sub-relato de sua massa corporal ${ }^{19}$. Além disso, aquelas que já haviam menstruado há mais de 3 anos tendiam a superestimar mais significantemente sua estatura. Da mesma forma que no atual estudo, no qual adolescentes que se classificavam como obesos apresentavam valores inferiores de IMC autorrelatados diante dos valores reais de IMC, é possível que as adolescentes australianas que já haviam menstruado há mais tempo fossem, consequentemente, mais baixas e, por isso, superestimassem sua altura em uma tentativa de se adequar ao padrão estético vigente.

Em estudo realizado com adolescentes brasileiros de 10 a 14 anos $(n=105)$, residentes em áreas rurais de Piedade $(\mathrm{SP})^{20}$, foi mostrado que as medidas autorreferidas de estatura e massa corporal apresentaram baixa concordância com as medidas aferidas em ambos os sexos. Na amostra em questão, a estatura foi subestimada entre meninos e meninas (média de -9,1 e - 7,5 cm, respectivamente), sendo as prevalências de sobrepeso e obesidade consequentemente superestimadas na população analisada. Os autores desse estudo enfatizam que essa grande discrepância pode ter sido observada devido à baixa frequência com que os moradores de áreas rurais costumam verificar suas medidas antropométricas. O presente estudo foi realizado em área urbana e apresentou, talvez devido a esse fato, dados bem diferentes do que os verificados no estudo realizado em Piedade ${ }^{20}$. Foi 
verificada, no atual estudo, alta concordância entre classificação de estatura para idade feita com base nos dados aferidos e referidos. Isso para a população adolescente chama bastante a atenção, pois se trata de uma faixa etária que está em fase de crescimento longitudinal, e, portanto, o valor dessa medida antropométrica está em constante mudança. Se os adolescentes desse grupo autorreferiram de forma adequada sua estatura, isso indica que esse grupo está aferindo de forma constante essa medida, talvez por uma preocupação estética na qual a estatura representa um valorizado parâmetro.

O estudo de Silveira et al. ${ }^{21}$, realizado com indivíduos adultos na zona urbana de Pelotas, município do Sul do Brasil ( $n=3.934$ ), buscou identificar as características socioeconômicas, demográficas e comportamentais de indivíduos que apresentam maior erro entre o IMC "referido" e o medido. Naquele estudo, renda familiar e idade foram variáveis associadas a erro de IMC referido, então os autores propuseram uma fórmula de correção para o IMC referido que utilizava as variáveis em questão. Em pesquisa realizada nos Estados Unidos com mais de 15.000 indivíduos ${ }^{22}$ na qual foi aplicada a correção do IMC referido, foi possível perceber que, mesmo corrigido, o IMC continua a subestimar a proporção de membros da população de obesos. No entanto, o IMC ajustado prevê estimativas precisas dos riscos de saúde associados a variações desse índice. Os autores concluem que os valores de IMC calculados com base na estatura e na massa corporal autorreferidos, se corrigidos para vieses característicos dos entrevistados, podem ser usados para fornecer estimativas precisas da proporção do excesso de peso nos membros da população.

Uma meta-análise publicada em $2009^{23}$, referente ao uso de medidas autorreferidas como massa corporal e estatura, citou apenas um estudo nacional sobre medidas autorreferidas em adolescentes. Trata-se do estudo realizado em Florianópolis ${ }^{14}$, já descrito neste trabalho. Os autores daquela meta-análise concluíram que, dentre os adolescentes, as medidas autorreferidas deveriam ser utilizadas com cautela uma vez que até aquele ano só havia um estudo desse tipo realizado com a população adolescente em território nacional e que, como a amostra daquele estudo era restrita a uma amostra local, poderia não representar a realidade das demais regiões brasileiras. A amostra do atual trabalho também se restringiu apenas a um grupo específico: adolescentes que estudavam na região Central de Cotia e, por essa razão, os resultados atuais também devem ser utilizados com cautela. Além disso, outra limitação do atual trabalho foi o fato de duas escolas públicas não terem aceitado participar do estudo, o que aumentou a porcentagem da amostra proveniente de escola particular (51\%). Isso faz com que a amostra do atual estudo não seja representativa dos adolescentes do município avaliado.
No atual estudo foi possível verificar que o sexo, a percepção da massa corporal e a imagem corporal foram variáveis que influenciaram na obtenção de respostas não válidas no que diz respeito aos dados antropométricos autorreferidos. Sendo assim, é importante se estar atento a essas variáveis, para que seja possível realizar uma análise mais robusta dos estudos realizados com adolescentes quando na obtenção de dados autorreferidos. Os dados relativos ao sexo e à percepção da massa corporal são dados simples de serem obtidos quando na tomada dos dados autorreferidos e, de acordo com Silveira et al. ${ }^{21}$, essas variáveis poderiam ser até mesmo utilizadas em estudos futuros para correção do IMC referido por meio de equações adequadas quando na impossibilidade de aferição das medidas visando à redução de eventuais discrepâncias entre as medidas referidas e aferidas.

De qualquer forma, é importante salientar que massa corporal e estatura não constituem medidas de difícil aferição e, considerando que possíveis erros podem acontecer durante o autorrelato, a aferição deve ser sempre estimulada. Essas medidas devem ser correta e regularmente aferidas nessa população, principalmente pelo aumento da prevalência de obesidade entre adolescentes e sua associação com agravos não transmissíveis, pois somente assim teremos condições de fornecer dados mais precisos para prevenir e combater precocemente essa doença.

\section{REFERÊNCIAS}

1. Marcondelli PDT, Marangon AFC. Peso e altura auto-referidos: um breve relato da literatura. Universitas. Ciências da Saúde (UNICEUB). 2005;3:161-71.

2. Pregnolato TS, Mesquita LM, Ferreira PG, Santos MM, Santos CC, Costa RF, et al. Validade de medidas autorreferidas de massa e estatura e seu impacto na estimativa do estado nutricional pelo índice de massa corporal. Rev Bras Crescimento Desenvolvimento Hum. 2009;19(1):35-41.

3. Peixoto MRG, Benício MHD, Jardim PCBV. Validade do peso e da altura autorreferidos: 0 estudo de Goiânia. Rev Saúde Pública. 2006;40(6):1065-72.

4. Branco LM. Relação entre o estado nutricional, a percepção e a satisfação da imagem corporal com a utilização de produtos diet e light, por adolescentes estudantes do ensino médio de uma escola pública do município de São Paulo [Dissertação de mestrado]. São Paulo: Programa de Pós-Graduação em Pediatria e Ciências Aplicadas à Pediatria, Universidade Federal de São Paulo; 2005.

5. Jelliffe DB. Evaluación directa del estado de nutrición de grupos humanos: Signos clínicos. In: Jelliffe DB, org. Evaluación del Estado de Nutrición de la Comunidad. Ginebra: Organización Mundial de la Salud, 1968. p. 10-53.

6. World Health Organization (WHO). Physical Status: the use and interpretation of anthropometry. Report of a WHO Expert Committee, No. 854, Geneva, 1965.

7. Madrigal-Fritsch H, Irala-Estevez J, Martinez-Gonzales MA, Kearney J, Gibney M, Martinez-Hernandez JA. Percepción de la imagen corporal como aproximación cualitativa al estado de nutrición. Salud Pública de México. 1999;41:479-86.

8. Cooper PJ, Taylor MJ, Cooper Z, Fairburn CG. The development and validation of the Body Shape Questionnaire. Int J Eating Disorder. 1987;6:485-94.

9. Cordás TA, Castilho S. Imagem corporal nos transtornos alimentares - instrumento de avaliação: "Body Shape Questionnaire". Psiquiatria Biológica. 1994;2:17-21.

10. Conti MA, Cordás TA, Latorre MRDO. Estudo de validade e confiabilidade da versão brasileira do Body Shape Questionnaire (BSQ) para adolescentes. Rev Bras Saúde Matern Infant. 2009;9(3):331-8. 
11. Streiner DL, Norman GR. Health Measurement Scales: A Practical Guide to Their Development and Use. 2nd ed. Oxford: Oxford University Press; 1995.

12. The SAS System for Windows (Statistical Analysis System), versão 8.02. SAS Institute Inc, 1999-2001, Cary, NC, USA.

13. Oliveira AGP, Oliveira TP, Ferreira MEC, Lima JRP. Diferença entre peso e estatura auto-referidos e aferidos para o cálculo do índice de massa corporal e sua relação com a imagem corporal de mulheres de academia de ginástica. HU Revista, Juiz de Fora. 2008;34(3):179-83.

14. Farias Jr JC. Validade das medidas auto-referidas de peso e estatura para 0 diagnóstico do estado nutricional de adolescentes. Rev Bras Saúde Matern Infant. 2007;7(2):167-74.

15. Brener ND, Mcmanus T, Galuska DA, Lowry R, Wechsler H. Reliability and validity of self-reported height and weight among high school students. J Adolesc Health. 2003;32(4):81-7.

16. Fonseca MJM, Faerstein E, Chor D, Lopes CS. Validade de peso e estatura informados e índice de massa corporal: estudo pró-saúde. Rev Saúde Pública. 2004;38:392-8.

17. Osuna-Ramirez I, Hernández-Prado B, Campuzano JC, Salmerón J. Indice de masa corporal y percepción de La imagen corporal en una población adulta mexicana: la precisión del autorreporte. Salud Publ Mex. 2006;48(2):94-103.
18. Niedhammer I, Bugel I, Bonenfant S, Goldberg M, Leclerc A. Validity of self-reported weight and height in the French GAZEL cohort. Int J Obes Relat Metab Disord. 2000;24:1111-8.

19. Abrahan S, Luscombe G, Boyd C, Olesen I. Predictors of the accuracy of self-reported height and weight in adolescent female school students. Int J Eat Disord. 2004;36:76-82.

20. Enes CC, Pegolo GE, Silva MV. Medidas autorreferidas versus medidas aferidas de peso e altura de adolescentes residentes em áreas rurais de Piedade, São Paulo. Rev Nutrire. 2009;34(2):59-70

21. Silveira EA, Araújo CL, Gigante DP, Barros AJD, Lima MS. Validação de peso e altura referidos para o diagnóstico do estado nutricional em uma população de adultos no Sul do Brasil. Cad Saúde Pública. 2005;21(1):235-45.

22. Stommel M, Schoenborn CA. Accuracy and usefulness of BMI measures based on self-reported weight and height: findings from the NHANES \& NHIS 2001-2006. BMC Public Health. 2009:9:421.

23. Coqueiro RS, Borges LJ, Araujo VC, Pelegrini A, Barbosa AR. Medidas auto-referidas são válidas para avaliação do estado nutricional na população brasileira? Rev Bras Cineantropom Desempenho Hum. 2009;11(1):113-9. 